\title{
Correction to: Highly electrically conductive and smart fire-resistant coating
}

\section{Bin Zhang ${ }^{1}$ (D) Yujie Jiang ${ }^{1}$}

Published online: 28 January 2019

○) Springer Science+Business Media, LLC, part of Springer Nature 2019

\section{Correction to: Journal of Materials Science: Materials in Electronics (2018) 29:16378-16387 https://doi.org/10.1007/s10854-018-9729-1}

The original version of the article unfortunately contained few errors in Figs. 1b, 1c, 4a, 4b and 7c. There are mismatches between published versions of these figures and the current pattern which we observed after repeated experiments. This erratum is published to correct them. The correct figures and reason for the corrections is given below.

In Fig. 1b, we found that the pure graphite is regular and single sheets and the Gr@PANI@Ag microcapsule (Fig. 1c) surface has no impurities and no white residue form in the surface. The revised images are shown here:
The original article can be found online at https://doi.org/10.1007/ s10854-018-9729-1.

\section{Bin Zhang}

zhangbin611@163.com

1 Zhejiang Provincial Key Laboratory of Industrial Textile Materials \& Manufacturing Technology, College of Materials and Textiles, Zhejiang Sci-Tech University, Hangzhou 310018, People's Republic of China
In Fig. 4a, the AP/Gr coated PP plastic is homogeneous and the particles are better distributed. Also, in Fig. 4b, the AP/Gr@PANI@Ag coated PP plastic surface is smooth and no fold is formed. The revised images are shown here:

In Fig. 7c, we found that the residual chars of pure PP have small holes and regular surface. After the observation of lots of particles in residual chars of AP coated PP plastic, we found that the particles are a better distribute. The residual chars of AP/6wt\%Gr@PANI@Ag coated PP plastic is smooth, no particles and folds are observed. Revised images are provided here: 
Fig. 1 b SEM micrograph of graphite; c SEM micrograph of Gr@PANI@Ag microcapsule

Fig. 4 a SEM images of AP/ Gr coated PP plastic; $b$ SEM images of AP/Gr@PANI@Ag coated PP plastic
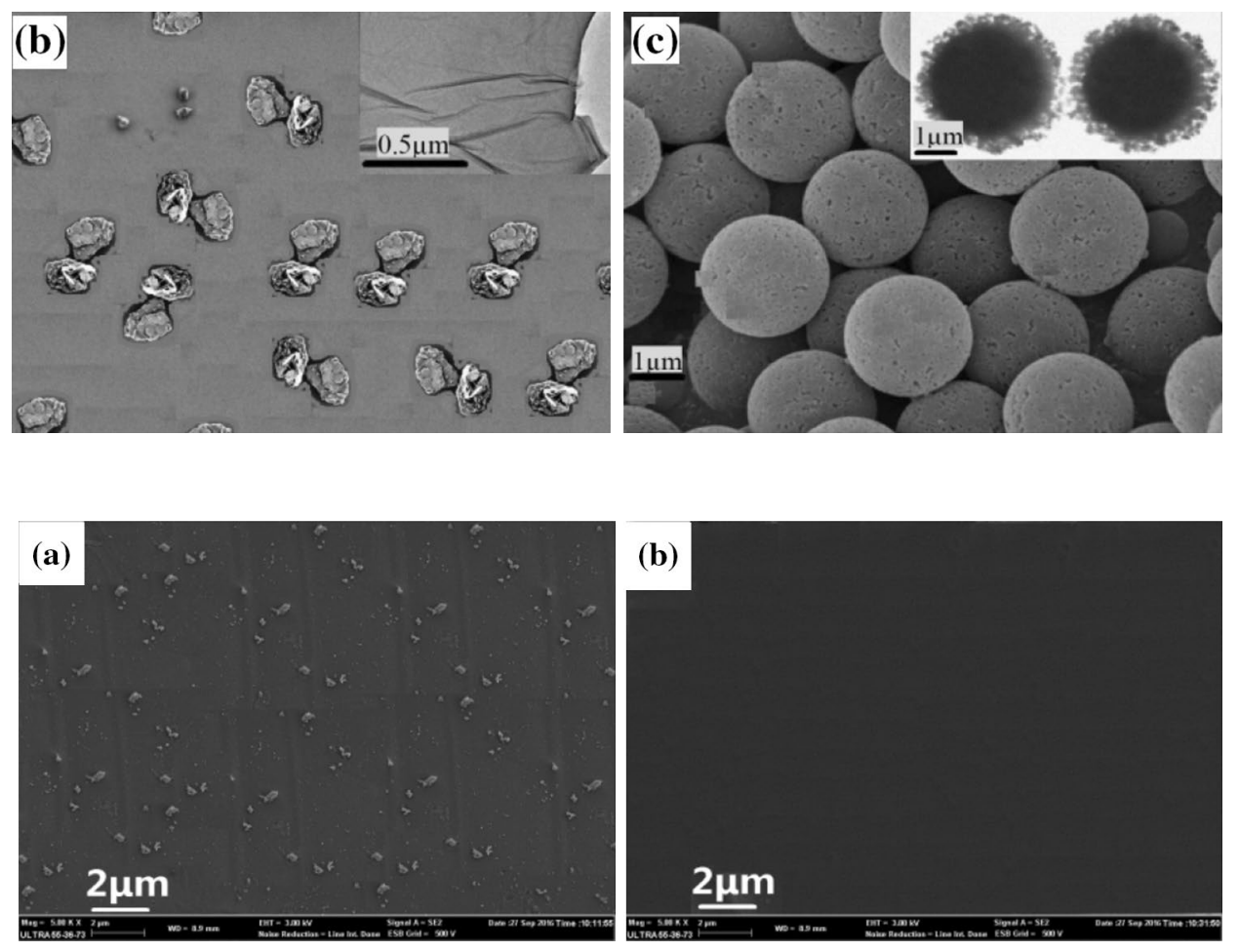

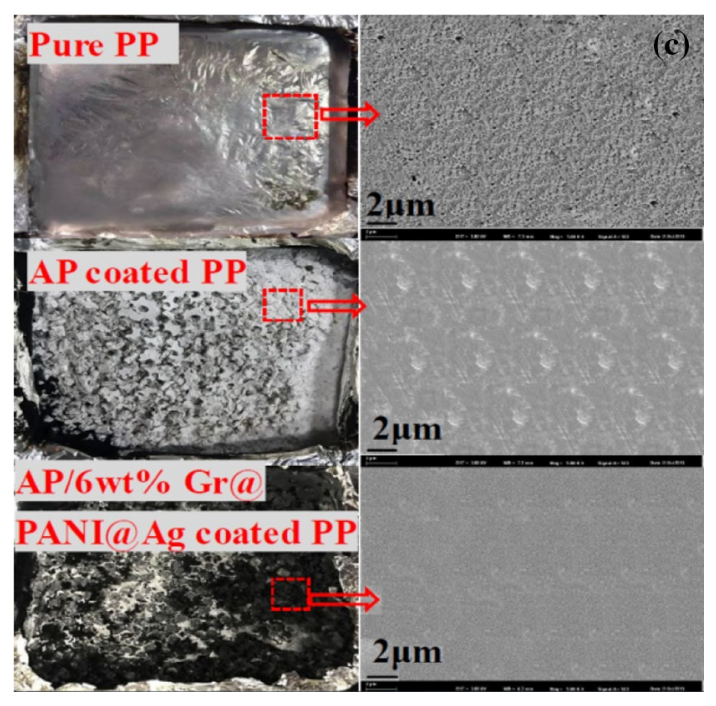

Fig. 7 c SEM of residual chars of pure PP, AP/Gr coated PP and AP/ Gr@PANI@Ag coated PP plastic 